DOI: $10.25100 /$ pfilosofica.v0i50.8701

\title{
PUNTOS, UNIDADES Y NÚMEROS: METAFÍSICA M.2 (1076B36-39)
}

\author{
José Edgar González-Varela \\ Instituto de Investigaciones Filosóficas, \\ Universidad Nacional Autónoma de México, \\ Ciudad de México, México.
}

\begin{abstract}
Resumen
En Metafísica M.2 Aristóteles desarrolla diversas objeciones en contra de la introducción platónica de objetos matemáticos como substancias nosensibles, separadas de las sensibles. Su primera objeción es de carácter doble. En primer lugar, Aristóteles argumenta que la postulación de objetos geométricos separados produce una acumulación absurda. En segundo lugar, sugiere que este argumento geométrico se puede extender al caso de las unidades y los números. En este trabajo me ocupo de explicar esta extensión aritmética. Los especialistas han interpretado esta extensión de una manera que denomino "maximalista". Aquí defiendo una interpretación distinta, "minimalista".
\end{abstract}

Palabras clave: anterioridad; objetos geométricos; números matemáticos; platonismo; separación.

Cómo citar este artículo: González-Varela, J. (2020). Puntos, unidades y números: Metafisica M.2 (1076b36-39). Praxis Filosófica, (50), 21-40. doi: 10.25100/pfilosofica.v0i50.8701.

Recibido: 28 de junio de 2019. Aprobado: 18 de septiembre de 2019. 


\title{
Points, Units and Numbers: Metaphysics M.2 (1076b36-39)
}

\section{José Edgar González-Varela ${ }^{1}$}

\begin{abstract}
In Metaphysics M.2 Aristotle develops several objections against the Platonist introduction of mathematical objects as non-sensible substances, separate from sensibles. His first objection has a two-fold nature. Firstly, Aristotle argues that positing separate geometrical objects produces an absurd accumulation. Secondly, he suggests that this geometrical argument can be extended to the case of units and numbers. In this paper I explain this arithmetical extension. Scholars have interpreted this extension in, what I call, a 'maximalist' way. Here I defend a different, 'minimalist', interpretation.
\end{abstract}

Keywords: Priority; Geometric Objects; Mathematical Numbers; Platonism; Separation.

${ }^{1}$ Investigador titular en el Instituto de Investigaciones Filosóficas de la Universidad Nacional Autónoma de México. Doctor en filosofía por la Universidad de Sheffield, Reino Unido. Sus áreas de especialización son la historia de la filosofía antigua, en particular la metafísica de Platón y Aristóteles, y la metafísica contemporánea. Ha publicado artículos sobre estos temas en revistas especializadas como Philosophy and Phenomenological Research, Phronesis, Apeiron, Revista Latinoamericana de Filosofía, Diánoia e Ideas y Valores.

ORCID: 0000-0003-1491-1844 E-mail: jvarela@filosoficas.unam.mx 


\title{
PUNTOS, UNIDADES Y NÚMEROS: METAFÍSICA M.2
} (1076B36-39)

\author{
José Edgar González-Varela \\ Instituto de Investigaciones Filosóficas, \\ Universidad Nacional Autónoma de México, \\ Ciudad de México, México.
}

\section{Introducción}

En los libros M-N de la Metafísica Aristóteles desarrolla una investigación de la propuesta platónica de que existen ciertas substancias aparte de las substancias sensibles, a saber, objetos matemáticos y Formas. En M.2 aborda la primera opción, los objetos matemáticos, discutiendo dos alternativas que los platónicos podrían seguir (pero sin identificar a ningún autor en particular): que los objetos matemáticos estén en las cosas sensibles o que estén separados ( $\kappa \varepsilon \omega \rho ı \mu \mu ́ v \alpha)$ de éstas (1076a33-35). Tras desechar rápidamente la primera alternativa, Aristóteles se concentra en la segunda. El primer argumento de Aristóteles es de carácter doble, en primer lugar, argumenta que la postulación de objetos geométricos separados produce una "acumulación absurda" (ó́o lugar, sugiere que este argumento geométrico se puede extender al caso de las unidades y los números (1076b36-39). Tanto el argumento geométrico como la extensión aritmética han sido, a mi juicio, sistemáticamente malinterpretados.

De acuerdo con el argumento geométrico de Aristóteles, la acumulación absurda que el platónico tiene que enfrentar consiste en que debe aceptar la existencia de un género de sólidos no-sensibles, pero tres de superficies,

${ }^{*}$ Este trabajo se realizó dentro del marco del proyecto PAPIIT IN403819, agradezco a la DGAPA (UNAM) por su generoso apoyo a mi investigación. 
cuatro de líneas y cinco de puntos no-sensibles (1076b29-33). En (GonzálezVarela, 2019) me ocupé de desarrollar una nueva interpretación de este argumento. El objetivo principal de esta interpretación era validar la cuenta final de Aristóteles, pues las interpretaciones disponibles de este argumento fallan en esta empresa: hacen que el argumento genere ya sea más o bien menos géneros de objetos geométricos ${ }^{2}$. Una de las claves para interpretar el argumento consiste en la identificación de una distinción implícita en éste entre acumulación cualitativa y acumulación cuantitativa. Una vez hecha esta distinción, y que se han entendido los criterios aristotélicos para que haya acumulación cualitativa, es posible apreciar que el argumento no puede generar más géneros de objetos geométricos de los que Aristóteles cuenta.

En este trabajo me ocupo de aprovechar los resultados de esta interpretación para ofrecer una interpretación de la extensión aritmética del argumento de Aristóteles. La interpretación que defiendo es una interpretación que podríamos denominar "minimalista", en tanto que sostiene que dicha extensión depende enteramente del argumento geométrico base y no añade nada más a la discusión, salvo la transformación del resultado geométrico al caso aritmético mediante una cierta hipótesis de conversión de puntos a números. De este modo, la acumulación de unidades y números que la extensión aritmética produce coincide exactamente con la acumulación de puntos que el argumento geométrico produce. Esta interpretación se opone a la interpretación "maximalista" que han defendido los intérpretes de M.2 desde, al menos, Pseudo-Alejandro. De acuerdo con ésta, la extensión aritmética no sólo aplica los resultados del argumento geométrico, sino que va más allá, generando una acumulación mayor: la acumulación de unidades y números no coincide con la de puntos, sino que es, incluso, potencialmente infinita. Aquí mostraré que esta interpretación maximalista carece de sustento. En la Sección 2 presento mi interpretación del argumento

\footnotetext{
${ }^{2}$ Por ejemplo, de acuerdo con la interpretación de Siriano (In Metaph. 87.6-9, Kroll), Pseudo-Filópono (p. 54, Lohr), Becker (1956, pp. 109-110), Annas (1976, pp. 140-141), Crubellier (1994, pp. 104-105) y Menn (2013, p. 22), el argumento geométrico produce 1 género de sólidos, 2 de superficies, 3 de líneas y 4 de puntos no-sensibles. De acuerdo con la interpretación de Ross (1924, Ar. Met., v. II, p. 413) y Apostle (1952, p. 201) éste produce 1 género de sólidos, 3 de superficies, 7 de líneas y 15 de puntos no-sensibles. De acuerdo con la interpretación de Katz (2014) produce 1 género de sólidos, 3 de superficies, 5 de líneas y 7 de puntos no-sensibles (cf. pp. 352-353, pero, especialmente, p. 354, n. 11). Pseudo-Alejandro (Hayduck, In Metaph. 726.35-727.40) es el único autor que ofrece una interpretación que valida la cuenta de Aristóteles, pero, como sostengo en (González-Varela, 2019), Pseudo-Alejandro malinterpreta claramente el texto para que le salga la cuenta. Robin (1908, p. 220) y Tricot (1953, v. II, p. 721) siguen la interpretación de Pseudo-Alejandro.
} 
geométrico y en la Sección 3 desarrollo mi interpretación de la extensión aritmética.

\section{El argumento geométrico}

El argumento de Aristóteles que afirma que la introducción platónica de objetos geométricos separados genera una acumulación absurda es el siguiente $(1076 \mathrm{~b} 11-36)^{3}$ :

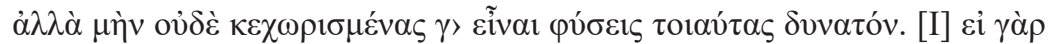

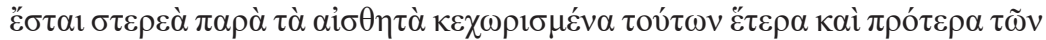

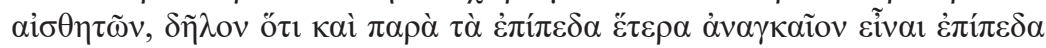

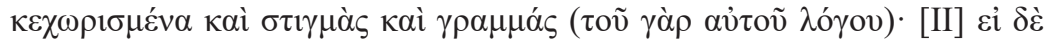

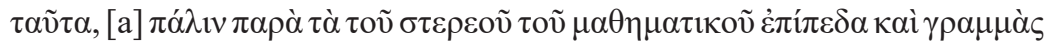

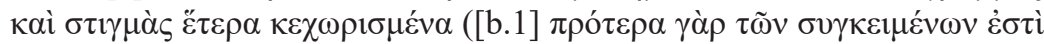

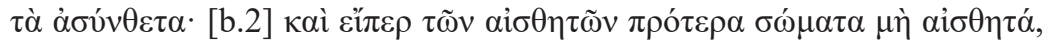

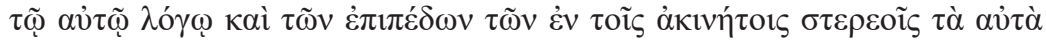

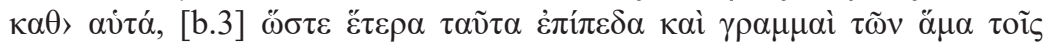

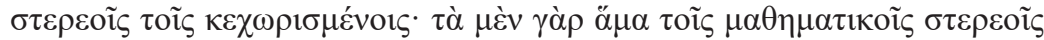

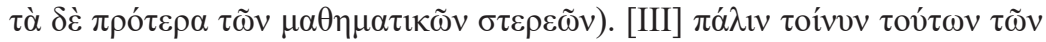

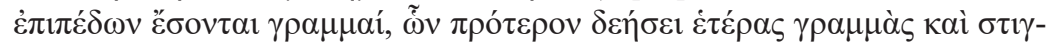

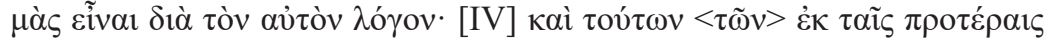

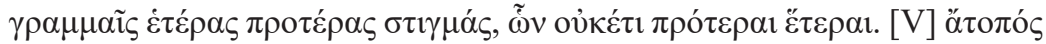

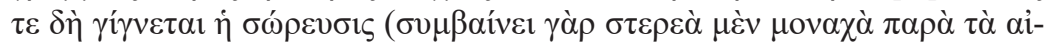

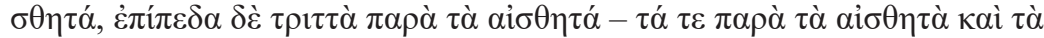

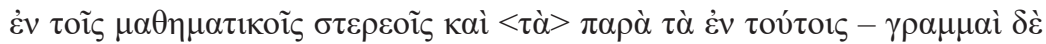

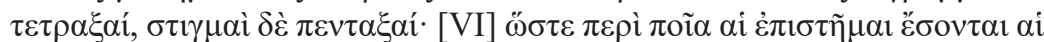

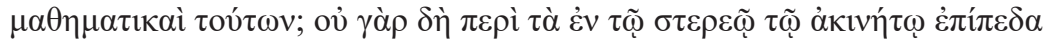

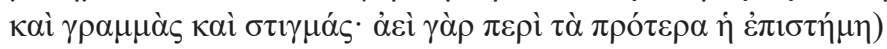

Pero tampoco es posible que estén separadas tales naturalezas. [I] Pues si ha de haber otros sólidos aparte de los [sólidos] sensibles, separados de y anteriores a los sensibles, es evidente que es necesario que también haya aparte de las superficies [sensibles] otras superficies separadas, y puntos y líneas (en efecto, por el mismo argumento). [II] Pero, si esto es así, [a] de nuevo, aparte de las superficies, líneas y puntos del sólido matemático [habrá] otros separados ([b.1] pues las cosas no combinadas son anteriores a las combinadas; [b.2] y si precisamente [existen] cuerpos no sensibles anteriores a los sensibles, por el mismo argumento también [existirán] aquellas [superficies] en sí y por sí [anteriores] a las superficies [que están] en los

${ }^{3}$ Todas las traducciones del griego en este trabajo son mías. El texto griego de la Metafísica que utilizo es el de Ross (Ar. Met., 1924). 
sólidos inmóviles; [b.3] de modo que estas superficies y líneas son distintas de las que están junto con los sólidos separados, pues las segundas están junto con los sólidos matemáticos, pero las primeras son anteriores a los sólidos matemáticos). [III] Entonces, de nuevo, habrá líneas de estas superficies, anteriores a las cuales será preciso que haya otras líneas y puntos por el mismo argumento. [IV] Y [será preciso que haya] otros puntos anteriores a aquellos [que están] en las líneas primeras, anteriores a los cuales ya no [habrá] otros. [V] La acumulación se vuelve ciertamente absurda (en efecto, resultan sólidos únicos aparte de los sensibles, pero triples superficies aparte de las sensibles - las [que son] aparte de las sensibles, las [que están] en los sólidos matemáticos y las [que son] aparte de las [que están] en éstos - y cuádruples líneas, y quíntuples puntos. [VI] De modo que ¿acerca de cuáles de éstos [objetos] serán las ciencias matemáticas? Pues no ciertamente acerca de las superficies, líneas y puntos [que están] en el sólido inmóvil, pues la ciencia siempre es acerca de las cosas primeras.)

El argumento de Aristóteles es complejo y de muy controvertida interpretación. Aquí me limitaré a esbozar sus líneas generales (para una justificación detallada de esta interpretación véase González-Varela, 2019). Primeramente, haré tres observaciones generales sobre el argumento.

(a) El argumento es una suerte de argumento regresivo que es tanto progresivo como decreciente. Es progresivo en el sentido de que introduce a cada paso objetos que son anteriores a los objetos del paso anterior, y es decreciente en el sentido de que introduce, también a cada paso, objetos menos complejos. Es decir, el argumento comienza introduciendo en [I] sólidos, superficies, líneas y puntos anteriores a los sensibles, y posteriormente introduce objetos anteriores y menos complejos hasta llegar, en [IV], a puntos anteriores a todos los demás. (b) El argumento utiliza dos nociones básicas, la de separación y la de anterioridad. Aristóteles distingue diferentes tipos de relaciones de separación y anterioridad (ontológica, definicional, etc.). En este argumento las relaciones de separación y anterioridad en cuestión son ontológicas ${ }^{4}$. No es necesario entrar aquí en detalle sobre la manera en que Aristóteles entiende las relaciones de separación y anterioridad en este argumento; es suficiente, por el momento, con notar que ambas consisten en una suerte de independencia ontológica. (c) La clave para entender el argumento radica en la distinción entre acumulación cualitativa y cuantitativa. Por acumulación cualitativa entiendo lo acumulación de distintos géneros, o clases, distintos de objetos geométricos. Por acumulación cuantitativa entiendo la acumulación de más objetos geométricos de un género antecedente ya reconocido. Es claro que

\footnotetext{
${ }^{4}$ Como sostiene también Katz (2014, p. 346; y 2017, pp. 52-57).
} 
Aristóteles intenta generar una acumulación cualitativa, no cuantitativa. El carácter progresivo del argumento es señal de esto: a cada paso Aristóteles se esfuerza por introducir objetos geométricos anteriores ontológicamente a los objetos precedentes. Y esto es así porque Aristóteles está entendiendo que podemos hablar realmente de objetos geométricos distintos unos de otros (v.gr., puntos distintos de puntos), si y sólo si, estos objetos geométricos se diferencian en sus relaciones de anterioridad. Es decir, Aristóteles asume que la pertenencia a distintos géneros está determinada, para los objetos matemáticos, por relaciones de anterioridad: $A$ pertenece a un género distinto de objeto matemático que $B$ si y sólo si $A$ es anterior ontológicamente a $B$ o $B$ es anterior ontológicamente a $A$. Como veremos, el argumento establece dos criterios de anterioridad (y separación) y es con base en éstos únicamente que se puede establecer la existencia de dos géneros distintos de objetos matemáticos. En el argumento geométrico Aristóteles ciertamente no utiliza el término "género" ( $\gamma \varepsilon ́ v o \varsigma)$ para indicar que está introduciendo una acumulación cualitativa, pero sí utiliza éste, como veremos, en la extensión aritmética. A continuación, explico brevemente cada paso del argumento.

Paso 1 (1076b12-16): Aquí Aristóteles introduce los objetos geométricos que los platónicos deben introducir para explicar las propiedades geométricas de los objetos sensibles, esto es, que hay sólidos, superficies, líneas y puntos sensibles. De acuerdo con Aristóteles, los platónicos deben introducir: (1) sólidos, superficies, líneas y puntos separados de los sensibles y anteriores a los sensibles. La idea es que dichos objetos matemáticos son separados de los sensibles y anteriores a los sensibles porque explican metafísicamente las propiedades geométricas de éstos, son su fundamento ontológico. Esta consideración, que es expresada por Aristóteles con la frase "el mismo argumento" ( de Separación" (SEP), la cual proporciona un principio importante de separación y anterioridad ontológica: el objeto geométrico $A$ que se dice separado y anterior con respecto a $B$ lo es en tanto que $A$ es el fundamento ontológico de $B$, mientras que $B$ no es el fundamento ontológico de $A^{5}$.

Paso II (1076b16-24): Aquí Aristóteles introduce dos géneros más de objetos geométricos. Primeramente [II.a], introduce: (2) "las superficies, líneas y puntos del sólido matemático", apelando a lo que denomino la "Premisa de Composición" (COMP), según la cual, los objetos geométricos separados (a excepción del punto) están compuestos de otros objetos geométricos de dimensiones inferiores. Asimismo, Aristóteles introduce: (3) “otros separados" y anteriores, es decir, otras superficies, líneas y puntos

${ }^{5}$ Para una interpretación similar de las nociones de separación y anterioridad ontológica en Aristóteles véase Corkum (2008 y 2016) y Katz (2017). 
separados y anteriores. En [II.b.1] (76b18-19) Aristóteles explica por qué los objetos (3) son anteriores a los objetos (2), porque los primeros son "no combinados" (ả $\sigma v ́ v \theta \varepsilon \tau \alpha)$, mientras que los segundos son "combinados"

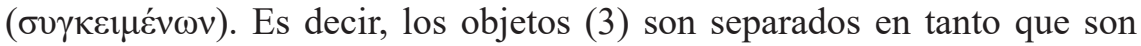
"por sí mismos", como se explica en [II.b.3], mientras que los objetos (2) son inmanentes o no-separados porque son los componentes de los sólidos matemáticos, como también se dice en [II.b.3]. Esta consideración introduce otro principio de separación y anterioridad, al cual, siguiendo a (Katz, 2014, p. 362), denomino (APS). De acuerdo con éste, si $A$ es por sí mismo y $B$ está en otra cosa, entonces $A$ es anterior ontológicamente a $B$.

(APS) y (SEP) son los únicos dos principios de separación y anterioridad empleados en el argumento, de los cuales se sirve Aristóteles para producir los distintos géneros de objetos geométricos. En efecto, (II.b.2) aclara que los objetos (3), superficies, líneas y puntos separados y anteriores, se introducen "por el mismo argumento" (76b20) que se introdujeron los sólidos matemáticos como separados y anteriores a los sensibles. Este argumento es, como vimos, (SEP). En consecuencia, la tesis enunciada en [II.b.3], que los objetos (3) "son anteriores a los sólidos matemáticos" (76b24), debe interpretarse de acuerdo con (SEP). Es decir, las superficies, líneas y puntos del grupo (3) son separados y anteriores a los objetos del grupo (1) porque explican metafísicamente sus propiedades geométricas, por ejemplo, explican cómo es que los sólidos matemáticos tienen superficies, líneas y puntos.

Paso III (1076b24-27): Aquí Aristóteles aplica de nuevo las premisas básicas (COMP), (SEP) y (APS). Primero, aplica (COMP), al mencionar a los componentes de las superficies del grupo (3), diciendo "habrá líneas de estas superficies" (b25), y, presumiblemente, también puntos. Luego dice que "será preciso que haya otras líneas y puntos anteriores a éstas" (b2526). Es decir, es necesario que haya, por (SEP), (4) otras líneas y puntos separados y anteriores que expliquen cómo es que las superficies del grupo (3) están compuestas de líneas y puntos. De nuevo, (SEP) se indica a través de la frase "el mismo argumento" (b26-27). Asimismo, estos objetos (4) son también separados y anteriores con respecto a las líneas y puntos que componen a las superficies del grupo (3) por (APS), porque los primeros son por sí mismos mientras que los segundos están en otro.

Paso IV (1076b27-29): Finalmente, Aristóteles aplica de nuevo las premisas (COMP), (SEP) y (APS) para generar el último grupo de objetos del argumento. Aristóteles habla de los puntos que están "en las líneas primeras" (76b27), es decir, aplica (COMP) para mencionar a los componentes inmanentes de las líneas del grupo (4). Y luego dice que será necesario que 
haya otros puntos anteriores (y separados). De nuevo, aunque aquí no se usa la frase "el mismo argumento", es claro que Aristóteles está aplicando (SEP) para producir el grupo de objetos (5), los puntos separados y anteriores que explican cómo es que las líneas del grupo (4) están compuestas de puntos.

El lector habrá notado que, si bien Aristóteles habla de dos grupos de líneas y puntos en el paso III, unos inmanentes y otros anteriores (y separados), y de dos grupos de puntos en el paso IV, unos inmanentes y otros anteriores (y separados), yo sólo he contado a los anteriores y separados dentro de la acumulación que produce el argumento. La razón de esto es que Aristóteles mismo no cuenta a los objetos inmanentes de los que habla en estos pasos. Pues, si los contara, entonces la cuenta final que hace unas cuantas líneas después (en [V]), a saber, que el argumento genera un género de sólidos, tres de superficies, cuatro de líneas y cinco de puntos sobre los sensibles, estaría patentemente equivocada. En efecto, si contara a estos objetos inmanentes habría cinco géneros de líneas y siete de puntos. ¿Por qué Aristóteles no cuenta a estos objetos? La explicación se halla en la distinción mencionada anteriormente entre acumulación cualitativa y cuantitativa. Aristóteles no cuenta a los objetos inmanentes de los pasos III y IV porque la introducción de éstos constituye sólo un caso de acumulación cuantitativa, no cualitativa. Esto es así porque, como vimos, la pertenencia a un género matemático está determinada por consideraciones de anterioridad: $A$ y $B$ pertenecen a géneros distintos si y sólo si $A$ es anterior a $B$ o $B$ es anterior a $A$. Y, de acuerdo con el argumento, sólo hay dos criterios válidos de anterioridad: (SEP) y (APS). Pero los objetos inmanentes de los pasos IV y V no pueden contar como géneros distintos por ninguno de estos dos criterios. Por un lado, no explican las propiedades geométricas de ningún objeto y, por ende, no son anteriores, por (SEP), con respecto a ninguno de los géneros de objetos introducidos en el argumento. Y, por (APS), ciertamente son posteriores a los objetos separados introducidos en el argumento, pero, crucialmente, no pueden, por este criterio, contar como anteriores o posteriores a los objetos geométricos del grupo (2), los componentes inmanentes de los sólidos matemáticos. En efecto, (APS) sólo establece que los objetos inmanentes son posteriores a los separados. Por ello, es razonable pensar que los objetos inmanentes que se mencionan en los pasos III y IV pertenecen al mismo género que los objetos inmanentes del grupo (2), es decir, todos tienen el mismo estatus ontológico y, por ello, Aristóteles no los cuenta, porque su adición representa sólo un caso de acumulación cuantitativa.

De este modo, mi interpretación valida plausiblemente la cuenta final que hace Aristóteles: 
Grupo 1: sólidos-1, superficies-1, líneas-1 y puntos-1 separados. Grupo 2: superficies-2, líneas-2, puntos-2 inmanentes.

Grupo 3: superficies-3, líneas-3, puntos-3 separados.

Grupo 4: líneas-4, puntos-4 separados.

Grupo 5: puntos-5 separados.

Ahora bien, Aristóteles piensa que esta acumulación de objetos geométricos es absurda porque genera una confusión en la concepción platónica de los objetos de la geometría. Aristóteles se pregunta en [VI] "¿acerca de cuáles de éstos [objetos] serán las ciencias matemáticas?", y piensa que los platónicos no tienen una respuesta satisfactoria. La aparentemente simple propuesta platónica de que los objetos del grupo (1) son los objetos de la ciencia geométrica se ve trastornada, pues hay más candidatos a ser los objetos de dicha ciencia. Aristóteles sugiere entonces que, en vista de este argumento, los platónicos deberían renunciar a la introducción de objetos geométricos separados.

En la siguiente sección analizaré en detalle la extensión que, de acuerdo con Aristóteles, es posible hacer del resultado de este argumento geométrico al caso de las unidades y números.

\section{La extensión aritmética}

Tras formular el argumento anterior en contra de la postulación platónica de objetos geométricos separados, Aristóteles sugiere que éste se puede extender también a los números matemáticos. Es decir, es posible extender el argumento geométrico para producir una acumulación absurda de números matemáticos. De este modo, si el argumento y la extensión son correctos, Aristóteles habrá mostrado que introducir cualquier objeto matemático separado es problemático. Esta extensión aritmética es sugerida por Aristóteles del siguiente modo (1076b36-39):

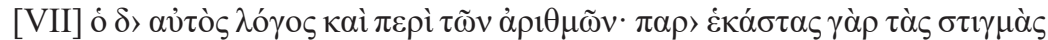

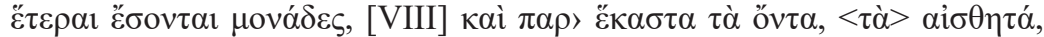

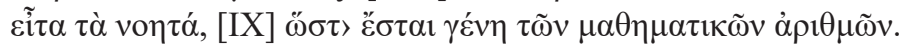

[VII] Pero el mismo argumento [se aplica] también a los números, pues habrá otras unidades aparte de cada uno de los puntos, [VIII] y aparte de cada uno de los entes, los sensibles, y a su vez los inteligibles, [IX] de modo que habrá géneros de los números matemáticos. 
A continuación, realizaré un análisis detallado de este argumento. Mi objetivo es defender, en contra de las interpretaciones maximalistas de esta extensión, una interpretación minimalista, según la cual, la acumulación de números producida por ésta depende totalmente de la acumulación producida por el argumento geométrico.

La extensión aritmética comienza en [VII] con la idea de que "el mismo argumento" se aplica a los números. Esta frase, en esta ocasión, no se refiere sólo a la premisa de separación (SEP), como en el argumento geométrico, sino a todo el argumento. Pues la idea es, evidentemente, que el resultado del argumento geométrico se puede extender a los números matemáticos. Dicha extensión la explica Aristóteles en la siguiente parte de [VII]. El "pues" ( $\gamma \alpha ́ \rho)$ inicial indica que ésta es, en efecto, una explicación de la frase anterior. La explicación de Aristóteles es muy breve, simplemente dice que "habrá otras

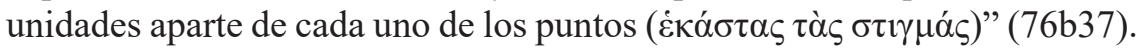
La extensión aritmética de Aristóteles intenta establecer, de este modo, una correspondencia uno a uno entre ciertos géneros de objetos geométricos, puntos, y géneros de unidades. La extensión parece depender entonces enteramente del argumento geométrico: habrá tantos géneros de unidades como géneros de puntos reconocidos en el argumento. Evidentemente, éste es un resultado indeseable para los platónicos, pues las unidades son, para ellos, la base de su filosofía de la aritmética. Si hubiera varios géneros de unidades, entonces se generaría del mismo modo una confusión en la ciencia aritmética, no habría una respuesta satisfactoria sobre cuáles son las unidades que constituyen la base de esta ciencia.

Pero ¿por qué piensa Aristóteles que si hay una acumulación no deseada de puntos la hay también de unidades? La razón de esto se basa en dos consideraciones. En primer lugar, Aristóteles supone que hay una cierta relación, para los platónicos, entre puntos y unidades. Un punto es la estructura espacial mínima, es absolutamente uno e indivisible. Luego entonces un punto es una cierta unidad, pero una unidad con posición. Las unidades matemáticas, en cambio, no son puntos, pues carecen de posición ${ }^{6}$. Aristóteles piensa entonces que los platónicos deben concluir la existencia de una unidad por cada punto que tengan que admitir en su ontología. La razón de esto no es afirmada explícitamente por Aristóteles, pero parece sugerida por la estructura misma de su explicación, la cual copia exactamente la manera como Aristóteles expresa, al menos en [I] y [II], la introducción de objetos geométricos anteriores y separados. En efecto, en [VII] Aristóteles

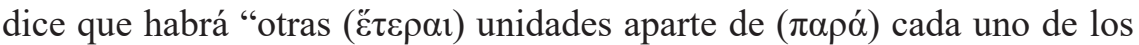

\footnotetext{
${ }^{6}$ Cf. Met. $\Delta .6$ 1016b24-31.
} 
puntos", de manera similar a como en [I] afirma que habrá "otros" ( $\check{\tau \varepsilon \rho \alpha) ~}$

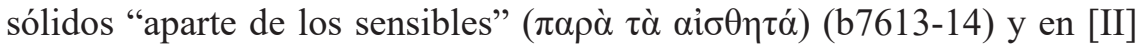
afirma que habrá "aparte de" ( $\pi \alpha \rho \alpha$ ) las superficies, líneas y puntos del

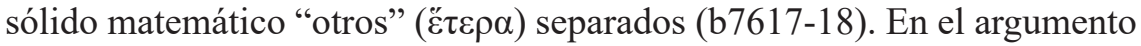
geométrico el término "otros" está acompañado por los calificativos "separados" o "anteriores", y es razonable pensar, dada la similitud de las frases, que en la extensión aritmética dichos términos se encuentran elididos. Es decir, parece razonable suponer que Aristóteles quiere decir que habrá otras unidades separadas y anteriores aparte de cada uno de los puntos. Como hemos visto, la razón por la cual los "otros" objetos geométricos son separados y anteriores a los precedentes es en virtud de la premisa (SEP), esto es, lo son porque explican metafísicamente que dichos objetos tengan las propiedades geométricas que tienen. Del mismo modo, entonces, Aristóteles supone que las unidades matemáticas sin posición son separadas y anteriores a los puntos correspondientes en virtud de que explican sus propiedades matemáticas, a saber que cada punto sea una unidad mínima.

Esta idea es clave en la extensión aritmética que propone Aristóteles, pues sólo de esta manera se explicaría por qué cada grupo de unidades correspondiente a cada grupo de puntos constituye un género distinto de unidad. Pues, recordemos, cada grupo de puntos es un género de punto distinto porque todos se distinguen en virtud de sus relaciones de anterioridad ontológica: cada grupo es anterior o posterior al otro. Esto es, cada grupo tiene un estatus ontológico distinto a cada otro grupo y, por ello, constituyen distintos géneros de puntos y conforman una acumulación cualitativa. Pero si cada grupo de unidades generado a partir de cada grupo de puntos es anterior al segundo, entonces también cada grupo de unidades es anterior a cada otro grupo de unidades. Es decir, los grupos de unidades parecen "heredar", por decirlo así, la estratificación ontológica de los puntos geométricos en virtud de la transitividad de la relación de anterioridad ontológica; del siguiente modo: si, por ejemplo, el grupo de puntos-5 es anterior ontológicamente al grupo de puntos- 4 , entonces, dado que el grupo de unidades- 5 es anterior al grupo de puntos-5 y el grupo de unidades-4 es anterior al grupo de puntos-4, entonces el grupo de unidades-5 es también anterior al grupo de unidades-4.

Sin embargo, este razonamiento deja todavía un hueco, pues podría objetarse que queda abierta la posibilidad de que las unidades-5 y las unidades-4 no sean anteriores ontológicamente unas a las otras, sino tan sólo anteriores a cada grupo de puntos. Por ello, me parece que la extensión de Aristóteles presupone también dos cosas más: que cualquier unidad es anterior ontológicamente a cualquier punto, y que las unidades conforman una escala ontológica distinta de los puntos. Es decir, los géneros de puntos 
tendrían un estatus ontológico con una escala de niveles de 1 a 5 , mientras que las unidades tendrían una escala ontológica de 6 en adelante. De modo que, si un grupo de puntos tiene un nivel de 4, entonces el grupo de unidades que explica a ese grupo de puntos tiene un nivel de $4+5$. Y si un grupo de puntos tiene un nivel de 5, entonces el grupo de unidades que explica a ese grupo de puntos tiene un nivel de $5+5$.

Ésta es, a mi juicio, la explicación más plausible de la extensión aritmética del argumento geométrico de Aristóteles. De acuerdo con ésta, la acumulación geométrica se extiende al caso aritmético: así como hay cinco géneros de puntos no-sensibles hay también cinco géneros de unidades no-sensibles. Es decir, las acumulaciones geométrica y aritmética se corresponden del siguiente modo, como se observa en la Tabla 1:

Tabla 1. Acumulación de puntos y unidades no-sensibles

\begin{tabular}{cc}
\hline Puntos & Unidades \\
\hline 1 & 1 \\
2 & 2 \\
3 & 3 \\
4 & 4 \\
5 & 5 \\
\hline
\end{tabular}

Sin embargo, la extensión aritmética de Aristóteles parece continuar en los pasos [VIII] y [IX], y es ahí en donde se localiza más propiamente la divergencia principal entre mi interpretación minimalista y las otras interpretaciones maximalistas.

Hay una controversia con respecto al texto de la parte [VIII]. El texto que he traducido aquí es el de Ross (1924, Ar. Met.), que incluye la adición

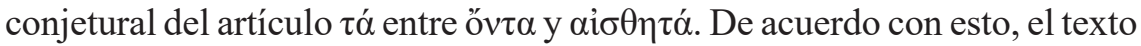

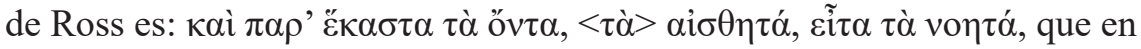
mi traducción dice: "y aparte de cada uno de los entes, los sensibles, y a su vez los inteligibles". Ross parece suponer que es necesario introducir este artículo para hacer a la frase simétrica. Es decir, sin el artículo el texto de los manuscritos dice "y aparte de cada uno de los entes sensibles, y a su vez los inteligibles". Es decir, Aristóteles en este caso hablaría descuidadamente primero de que habría otras unidades aparte de cada uno de los géneros de entes sensibles y después se corregiría diciendo que también habría otras unidades aparte de cada uno de los entes inteligibles. En cambio, con la conjetura de Ross, Aristóteles afirma al principio que habrá otras unidades con respecto a cada uno de los géneros de entes (relevantes), y después aclara, 
simétricamente, que las habrá tanto en el caso de los entes sensibles como en el de los entes inteligibles. La mayoría de los intérpretes posteriores acepta la conjetura de Ross, pero, como se verá a continuación, la interpretación minimalista que propongo requiere de dicha conjetura, mientras que las interpretaciones maximalistas pueden leerse tanto con ella como sin ella ${ }^{7}$. Sin embargo, al mostrar que la interpretación maximalista carece de sustento mostraré también que la conjetura de Ross es necesaria no sólo por razones estilísticas sino de contenido.

De acuerdo con las interpretaciones maximalistas de la extensión aritmética que se han ofrecido, desde Pseudo-Alejandro, la parte [VIII] añade algo que no depende en sentido estricto del argumento geométrico. Es decir, produce una acumulación mayor de unidades (y números) que la acumulación de puntos que produce el argumento geométrico. En efecto, de acuerdo con estas interpretaciones, őv $\tau \alpha$ incluye a todos los entes por igual, sensibles o inteligibles. Es decir, Aristóteles se está refiriendo aquí a todos los entes sensibles, como los perros, limones o mesas, y a todos los entes inteligibles, como las Ideas, las almas, los teoremas matemáticos, etc. En consecuencia, lo que dice Aristóteles en [VIII] es que no sólo habrá un género de unidades distinto por cada género de puntos del argumento geométrico, sino, además, que habrá un género de unidad distinto por cada género de ente, tanto sensibles como inteligibles. Así pues, habrá tantos géneros de unidad como géneros de ente. Pero, como parece haber realmente muchos géneros de entes sensibles e inteligibles, entonces habrá muchos géneros de unidades, no sólo los que corresponden a los puntos ${ }^{8}$.

Ahora bien, la parte [IX] del texto dice: "de modo que habrá géneros de los números matemáticos". Primeramente, es necesario aclarar por qué Aristóteles dice que habrá "géneros" de los números y no sólo de las

${ }^{7}$ La aceptan, por ejemplo, Jaeger (1957), Annas (1976), García Yebra (1982), Calvo Martínez (2000) y Reale (2004). Crubellier (1994, p. 108), por su parte, piensa que no es necesario introducir esta conjetura, pues no cree que haya ningún problema en suponer que la frase es asimétrica inicialmente y luego Aristóteles rectifica.

${ }^{8}$ Ejemplos claros de esta interpretación maximalista son los siguientes. Annas (1976) explica el texto [VIII] de la siguiente manera: "Aristotle extends the argument beyond different types of points to difference in type of any object counted; and this move is independent of the last argument" (p. 142, el énfasis es mío). Crubellier (1994), por su parte, lo explica así: "A ces quatre types d'unités correspondant aux classes de points, s'en ajoute, non pas un, mais deux autres: car on peut dénombrer, non seulement les objets sensibles (les oies ou les bateaux), mais aussi les objets intelligibles (les solutions d'une équation, ou les vertus cardinales), et c'est en cela que l'argument sur les nombres se distingue de celui des objets géométriques" (p. 108, mi énfasis). Esta interpretación se remonta, al menos, a PseudoAlejandro (Hayduck, In Metaph. 728.12-22). Otros autores que la aceptan son, por ejemplo, Apostle (1966, p. 411, n. 14) y Reale (2004, p. 1303). 
unidades. La razón es que, para los antiguos, los números son pluralidades de unidades. Por ejemplo, el número 5 es una pluralidad de cinco unidades ${ }^{9}$. Luego entonces, en [IX] Aristóteles efectúa una extensión que parece, a la luz de la aritmética antigua, razonable: si hay géneros de unidades, entonces también habrá géneros de números, porque los números son pluralidades de unidades. De este modo, la idea es que por cada género de unidad habrá un género de número. Sin embargo, algunos defensores de la interpretación maximalista han pretendido reflejar su interpretación también en esta parte. Por ejemplo, Jaeger (1957) propone en su edición del texto la adición conjetural de un ǒ $\pi \varepsilon \iota \rho \alpha$, "infinitos" o "ilimitados", entre ع̌ $\sigma \tau \alpha \iota$

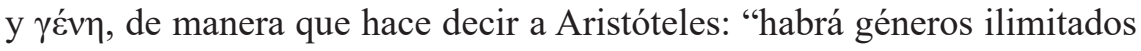
de los números matemáticos". En esto sigue la paráfrasis o explicación que Pseudo-Alejandro, (Hayduck, In Metaph. 728.22-23) hace de la extensión aritmética. Aunque no todos los intérpretes que defienden una interpretación maximalista aceptan esta adición, es claro que la razón por la que la rechazan no es porque sea incorrecta, sino porque, a su juicio, es innecesaria. En efecto, la adición de Jaeger refleja perfectamente la interpretación maximalista. Pues, si de acuerdo con [VIII], hay un género de unidades por cada género de entes, tanto sensibles como inteligibles, entonces habrá muchísimos ilimitados géneros de unidades $\mathrm{y}$, en consecuencia, ilimitados géneros de números ${ }^{10}$.

Sin embargo, la interpretación maximalista distorsiona la extensión aritmética de Aristóteles y hace que ésta quede, en su parte [VIII], sin

${ }^{9}$ Cf. Euclides, Elementos, Libro VII, definición 2, en Heath (1908).

${ }^{10}$ La conjetura de Jaeger (1957) es aceptada, por ejemplo, por Apostle (1966), Annas (1976), Calvo Martínez (2000) y Reale (2004). Annas (1976) expresa bien la idea detrás de esta conjetura, Aunque ella dice que el argumento está completo sin esta inserción, añade que: "This insertion is probably right however ... [the extension] ... does not show anything absurd in the platononist's position, unless 'infinitely many' is added" (p. 142). Crubellier (1994), por su parte, difiere de Annas; no cree que sea necesario incluir la conjetura para que la extensión aritmética muestre algo absurdo: "On voit d'après la note précédente que l'addition de $\alpha \varkappa \pi \varepsilon i \rho \alpha$ après $\gamma \varepsilon ́ v \eta$ est inutile; le point important est qu'il y aura des genres différents, de nombre mathématique, ce qui est contraire à la conception même du nombre mathématique" (p. 108). En esto tiene enteramente razón. La acumulación es absurda, como vimos, simplemente porque es cualitativa, porque genera varios géneros de números. Sin embargo, aunque Crubellier piensa que la adición es inútil, no sugiere que sea incorrecta. $\mathrm{Si}$ bien en el párrafo citado en la nota 7 Crubellier parece sugerir que sólo habría dos géneros más de unidades, "s'en ajoute, non pas un, mais deux autres" tipos de unidades (p. 108), en su esquema inicial de M.2 afirma que "il y aura une classe d'unités pour chaque classe de points géométriques, mais aussi pour les classes d'objets sensibles et de même pour les intelligibles" (p. 89). Es decir, el plural "classes" parece sugerir que habrá tantos géneros de unidad como clases de objetos sensibles e inteligibles. Ésta es, en cualquier caso, la interpretación más plausible del texto si se rechaza la inserción de Ross de un tó en b38, como hace Crubellier. 
ningún sustento. En efecto, como vimos en la sección anterior, el objetivo de Aristóteles al formular su argumento geométrico es generar una acumulación cualitativa de objetos geométricos, es decir, una acumulación de géneros de objetos geométricos. Y, como advertimos, la pertenencia a un género geométrico está determinada por relaciones de anterioridad ontológica: $A$ pertenece a un género distinto de $B$ si y sólo si $A$ es anterior a $B$ o $B$ es anterior a $A$. En efecto, sólo en ese caso estaría justificado decir que, por ejemplo, los puntos introducidos en el paso [IV] son genuinamente distintos de los puntos introducidos en el paso [I]: porque unos son anteriores ontológicamente a los otros. Pero parece plausible que, si Aristóteles piensa que "el mismo argumento" se aplica a las unidades y los números, esto es porque dicha aplicación aritmética debería producir una acumulación cualitativa de unidades y números, es decir, debería producir géneros de unidades y de números. He explicado como ocurre esto si suponemos, como parece hacerse en [VII], que la extensión aritmética consiste sólo en producir tantos géneros de unidades como géneros de puntos: los grupos correspondientes de unidades heredan su ordenamiento por relaciones de anterioridad del ordenamiento correspondiente de los puntos. Por ello es por lo que podemos decir que los cinco grupos de unidades que se obtienen a partir de los cinco grupos de puntos son genuinamente géneros distintos de unidades, porque cada grupo es anterior o posterior ontológicamente a cada otro.

Pero, si suponemos, con la interpretación maximalista, que la parte [VIII] pretende ir más allá y generar más géneros de unidades, esta extensión adicional carecería enteramente de sustento. No habría ninguna razón pare pensar que los grupos adicionales de unidades generados son realmente géneros distintos, pues no se diferenciarían por las relaciones de anterioridad ontológica existentes entre ellos. Y esto es así porque los grupos de objetos a partir de los cuales se obtendrían las unidades no están estratificados todos ellos mediante relaciones de anterioridad ontológica. De modo que las unidades generadas no pueden heredar dichas relaciones de éstos. Por ejemplo, los perros y los gatos no están estratificados, es decir, unos no son anteriores ontológicamente a otros. Similarmente, las almas y los puntos no están estratificados, es decir, unos no son anteriores ontológicamente a otros. Luego entonces, las unidades obtenidas a partir de estos grupos no podrían heredar su estratificación ontológica a partir de ellos y, por ende, no habría ninguna razón para pensar que las unidades obtenidas a partir de los gatos serían distintas de las unidades obtenidas a partir de los perros, o que las unidades obtenidas a partir de las almas serían distintas de las unidades obtenidas a partir de los puntos. Y tampoco hay razones independientes para pensar que los respectivos grupos de unidades estarían 
ordenados por relaciones de anterioridad ontológica. Por ende, la supuesta extensión aritmética adicional efectuada en [VIII] fallaría claramente, no habría razones para pensar que haya un género distinto de unidades por cada género de entes ${ }^{11}$.

Por estas razones, considero que las interpretaciones maximalistas, que pretenden que [VIII] intenta producir una acumulación mayor de unidades que la acumulación de puntos producida por el argumento geométrico, están equivocadas. ¿Cómo debemos entonces entender el texto [VIII]? Propongo que entendamos su función como enteramente aclaratoria: el texto no añade ningún argumento o consideración nueva. Es decir, propongo que interpretemos que la función del кaí inicial es epexegética, y que, por tanto, el texto debe entenderse de la siguiente forma: "esto es, aparte de cada uno de los entes, los sensibles, y a su vez los inteligibles". En mi interpretación, entonces, la referencia de ő $v \tau \alpha$ debe restringirse a los entes relevantes de los que se ha hablado en [VII], es decir, puntos. Lo que quiere decir Aristóteles entonces es: habrá un género de unidades por cada género de puntos, esto es, por cada género de entes relevante (puntos), los puntos sensibles, y, a su vez, los puntos inteligibles. Es decir, [VIII] aclara que la extensión aritmética abarca no sólo a los puntos inteligibles, sino también a los sensibles. Pues, como Aristóteles expresa claramente en [I], no sólo existen los cinco géneros de puntos inteligibles que su argumento genera, sino también los puntos sensibles a partir de los cuales los platónicos postulan los puntos inteligibles separados iniciales. Así, de acuerdo con la extensión aritmética de Aristóteles, hay tantos géneros de unidades y de números como géneros de puntos (sensibles e inteligibles), tal y como se muestra en la Tabla 2:

Tabla 2. La extensión aritmética completa

\begin{tabular}{ccc}
\hline Puntos sensibles & Unidades sensibles & Números sensibles \\
\hline 1 & 1 & 1 \\
2 & 2 & 2 \\
3 & 3 & 3 \\
4 & 4 & 4 \\
5 & 5 & 5 \\
\hline
\end{tabular}

${ }^{11}$ Es posible que en algunos casos haya razones para pensar que algunos objetos base son anteriores ontológicamente a otros. Por ejemplo, las almas son anteriores, para los platónicos, a los cuerpos. Sin embargo, mi objeción es que no hay una razón de principio para sostener que las unidades pueden heredar sus relaciones de anterioridad ontológica de la de los objetos base. Y hay, además, muchos casos en los que no se puede afirmar tal anterioridad ontológica, como en los casos mencionados de perros y gatos, por un lado, y almas y puntos, por el otro. 
Éstos son, así, los únicos géneros de unidades y de números que Aristóteles puede generar legítimamente. Los cinco géneros de números no-sensibles son genuinamente distintos porque heredan sus relaciones de anterioridad ontológica de las relaciones correspondientes de las unidades no-sensibles, las cuales las heredan, a su vez, de las relaciones de los puntos. Vemos entonces que la acumulación (cualitativa) de unidades y números depende enteramente de la acumulación (cualitativa) de puntos generada en el argumento geométrico. Por tanto, las interpretaciones maximalistas que pretender producir una acumulación mayor (ilimitada) de unidades y números independientemente del argumento geométrico están equivocadas. Y esto me parece razonable, pues a final de cuentas Aristóteles dice que "el mismo argumento" se aplica a los números.

\section{Referencias bibliográficas}

Annas, J. (1976). Aristotle's Metaphysics, Books $M$ and N. Oxford, Inglaterra: Clarendon Press.

Apostle, H. G. (1952). Aristotle's Philosophy of Mathematics. Chicago, EUA: Chicago University Press.

Apostle, H. G. (Trad.). (1966). Aristotle’s Metaphysics. Bloomington, EUA: Indiana University Press.

Becker, O. (1956). Formallogisches und Mathematisches in griechischen philosophischen Texten. Philologus, 100(1-2), 108-112. doi: 10.1524/ phil.1956.100.12.108.

Calvo Martínez, T. (Trad.). (2000). Aristóteles. Metafisica. Madrid, España: Gredos. Corkum, P. (2008). Aristotle on Ontological Dependence. Phronesis, 53(1), 65-92. doi: $10.1163 / 156852808 X 252594$.

Corkum, P. (2016). Ontological Dependence and Grounding in Aristotle. Oxford Handbooks Online. doi: 10.1093/oxfordhb/9780199935314.013.31.

Crubellier, M. (1994). Les livres "Mu" et "Nu" de la Métaphysique d'Aristote: traduction et commentaire (tesis doctoral). Université Charles De Gaulle LilleIII, Villeneuve d'Ascq, Francia.

García Yebra, V. (Trad.). (1982). Metafisica de Aristóteles. Madrid, España: Gredos. González-Varela, J. E. (2019). The Platonist Absurd Accumulation of Geometrical Objects: Metaphysics M.2. Phronesis, advance article, 1-40. doi: 10.1163/15685284-12342074.

Hayduck, M. (Ed.). (1881). Commentaria in Aristotelem Graeca 1: 1, Alexander Aphrodisiensis. In Aristotelis Metaphysica commentaria. Berlín, Alemania: Reimer.

Heath, T. L. (1908). Euclid, The Thirteen Books of the Elements. Vol. 2 (Books III$I X)$. Cambridge, Inglaterra: Cambridge University Press.

Jaeger, W. (Ed.). (1957). Aristotelis Metaphysica. Oxford, Inglaterra: Clarendon Press. 
Katz, E. (2014). An Absurd Accumulation: Metaphysics M.2, 1076b11-36. Phronesis, 59(4), 343-368. doi: 10.1163/15685284-12341272.

Katz, E. (2017). Ontological Separation in Aristotle's Metaphysics. Phronesis, 62(1), 26-68. doi: 10.1163/15685284-12341318.

Kroll, W. (Ed.). (1902). Commentaria in Aristotelem Graeca 6: 1, Syrianus. In Metaphysica commentaria. Berlín, Alemania: Reimer.

Lohr, Ch. (Ed.). (1991). Pseudo-Philoponus. Expositiones in omnes XIV libros Metaphysicos. Stuttgart-Bad Cannstatt, Alemania: Frommann-Holzboog.

Menn, S. (Ed.). (2013). Ig3: 'The Argument Structure of MN and their Place in the Metaphysics'. En The Aim and the Argument of Aristotle's Metaphysics (versión preliminar). Berlín, Alemania: Humboldt-Univerzität zu Berlin. Recuperado de https://www.philosophie.hu-berlin.de/de/lehrbereiche/antike/mitarbeiter/ menn/texte/ig3.

Reale, G. (2004). Metafisica di Aristotele. Milán, Italia: Bompiani.

Robin, L. (1908). La théorie platonicienne des idées et des nombres d'après Aristote. París, Francia: Félix Alcan.

Ross, W. D. (Ed.). (1924). Aristotle's Metaphysics. 2 vols. Oxford, Inglaterra: Clarendon Press.

Tricot, J. (Tr.). (1953). Aristote, La métaphysique. París, Francia: Vrin. 
